\title{
Validation of HPLC and FIA Spectrophotometric Methods for the Determination of Lansoprazole in Pharmaceutical Dosage Forms and Human Plasma
}

\author{
Idrees F. Al-Momani*, Majdoleen H. Rababah \\ Chemistry Department, Yarmouk University, Irbid, Jordan \\ E-mail:imomani2000@yahoo.com \\ Received January 10, 2009; revised February 21, 2009; accepted February 23, 2009
}

\begin{abstract}
A chromatographic and aspectrophotometric methods for the quantitative determination of lansoprazole in pharmaceutical combinations and human plasma were developed. The analytical parameters were studied according to International Conference on Harmonization guidelines. The Flow Injection Analysis (FIA) method is based on the oxidation of lansoprazole by a known excess of N-bromosuccinimide (NBS) in an acidic medium, followed by a reaction of excess oxidant with chloranilic acid (CAA) to bleach its purple color. The separation was carried out using RP-C18 column with a mobile phase composed of ACN: TEA: phosphate buffer (60: 0.2: $39.8 \mathrm{v} / \mathrm{v})$ adjusted to $\mathrm{pH}=4$.
\end{abstract}

Keywords: Lansoprazole, FIA, HPLC, Pharmaceutical Products, Human Plasma

\section{Introduction}

Lansoprazole, 2-[[[3-methyl-4-(2,2,2-trifluoroethoxy)-2pyridinyl]methyl] sulfinyl]-1H-benzimidazole, is an important proton pump inhibitor that suppresses gastric acid secretion by specific inhibition of the gastric $\mathrm{H}^{+}, \mathrm{K}^{+}$ ATPase enzyme system. Lansoprazole is effective in the treatment of various peptic diseases, including gastric and duodenal ulcer and reflux esophagitis, ZollingerEllison syndrome [1]. Proton pump inhibitors are unstable at a low $\mathrm{pH}$, therefore, the oral dosage forms are supplied as enteric-coated granules encapsulated in a gelatin shell. Owing to the vital importance of the drug, the development of sensitive, simple and fast methods for its determination is of urgent need. [1,2].

A survey of literature has revealed several analytical methods for the determination of lansoprazole in biological fluids and in pharmaceutical products. These include; high-performance liquid chromatography (HPLC) [3-7], electrochemical [8-10] and spectrophotometric methods [11-13]. The literature reports few spectrophotometric methods for the quantitation of lansoprazole. The reported methods need heating and long time for the color formation. According to our knowledge, only one FIA method was reported in the literature for the determination of lansoprazole. In addition, some of the reported HPLC methods need sophisticated instruments or do not describe analytical parameters that are very important for the validation of analytical procedure such as accuracy, specificity, robustness, limit of detection (LOD) and limit of quantitation (LOQ).

Since lansoprazole is acid labile, it is important to develop and validate analytical methods for its determination in the presence of its degradation products. The HPLC method has been highly used in the quality control of drugs because of their sensitivity, reproducibility and specificity. The FIA spectrophotometric method is very simple, rapid and economical and allows the determination of drugs with sufficient reliability. The present work reports the development and validation of a FIA and HPLC methods for the estimation of lansoprazole in pharmaceutical products and in human plasma.

\section{Experimental}

\subsection{Chemicals}

All chemical used were in pure grade and used as received without further purification. The active ingredient lansoprazole was supplied by the United Pharmaceuticals. All pharmaceutical products used in this work were purchased from the local market. Acetonitrile (ACN) and methanol were HPLC grade and obtained from LABSCAN. Triethylamine (TEA) was obtained from Cam- 
brian Chemicals. Phosphoric acid, N-bromosuccinimid and Chloranilic acid were obtained from Fluka Chemicals Ltd. Ultrapure water was used to prepare all solutions. All solutions were prepared daily.

\subsection{Instrumentation and Analytical Conditions}

The FIA system was assembled from $0.51 \mathrm{~mm}$ micro line tubes. This manifold consists of three microline tubes (Figure 1). The first tube contains the carrier $(0.2 \mathrm{M} \mathrm{HCl})$ which carries the sample toward the first reaction coil in order to react with the reagent (NBS) which is pumped in the second tube. The CAA stream was then added at a rate of $1.0 \mathrm{~mL} / \mathrm{min}$ in a confluence manner down stream to ensure rapid and adequate mixing. The excess NBS was then allowed to react with the CAA in the second reaction coil ( $\mathrm{RC} 2)$. After injection, the valve was returned to the load position when the maximum change in absorbance value has been reached. The absorbance of the remaining CAA was monitored at $530 \mathrm{~nm}$ by a Varian DMS-100 UV-Visible spectrophotometer equipped with an $18 \mu \mathrm{L}$ flow cell.

The HPLC method was performed on a Knauer model501 liquid chromatograph system. The system is equipped with a $20 \mu \mathrm{L}$ manual injector, $10 \mathrm{~mL}$ ceramic head pump and a programmable variable wavelength UV detector. Eurochrom software was used for all chromatographic measurements. A reversed-phase column $(15 \mathrm{~cm} \times 4.0$ mm ID., $5 \mu \mathrm{m}$ particle size, C18 column) was used at ambient temperature. Lansoprazole was eluted isocratically with a flow rate of $0.5 \mathrm{~mL} / \mathrm{min}$ and monitored at $285 \mathrm{~nm}$. The mobile phase was prepared by using ACN: TEA:phosphate buffer $(60: 0.2: 39.8, \mathrm{v} / \mathrm{v})$ adjusted to $\mathrm{pH}$ $=4$ using phosphoric acid. The mobile phase was filtered using $0.45 \mu \mathrm{m}$ PTFE filters, stirred and degassed before use.

\subsection{Preparation of the Standard Solutions}

1) N-bromosuccinimid (NBS): Accurately weighed 44.5 mg of NBS was transferred to $250 \mathrm{~mL}$ volumetric flask and dissolved in $0.1 \mathrm{M} \mathrm{HCl}$. The final concentration was

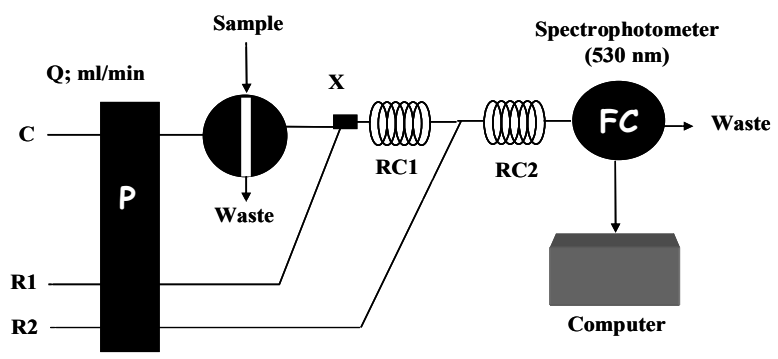

Figure (1): Schematic diagram of the proposed FIA system. $P$, peristaltic pump; $X$, confluence point; $C$, carrier solution, 0.2 M HCl; R1, $1.0 \mathrm{mM}$ NBS; R2, $1.0 \mathrm{mM} \mathrm{CAA;} \mathrm{RC1} \mathrm{and}$ $\mathrm{RC} 2$ are the reaction coils; $\mathrm{FC}$, flow cell.
$1.0 \mathrm{mM}$.

2) Chloranilic acid (CAA): Accurately weighed 52.0 $\mathrm{mg}$ of CAA was transferred to $250 \mathrm{~mL}$ volumetric flask and dissolved in ultrapure water. The final concentration was $1.0 \mathrm{mM}$.

3) Lansoprazole standard solutions: Accurately weighed $100.0 \mathrm{mg}$ of lansoprazole reference standard was transferred to $100 \mathrm{~mL}$ volumetric flask and dissolved in $0.1 \mathrm{M}$ $\mathrm{HCl}$. Standard solutions for linearity study were prepared by diluting the calculated volumes of the stock solution with $0.1 \mathrm{M} \mathrm{HCl}$.

4) Internal standard: The internal standard (IS) solution was prepared by dissolving $12.5 \mathrm{~g}$ of xanthon in 250 $\mathrm{mL}$ of methanol. The final concentration was $50 \mu \mathrm{g} / \mathrm{mL}$. The solution was then used as a solvent to prepare all other solutions to be analyzed by HPLC.

\subsection{Preparation of the Sample Solutions}

1) Capsules: The contents of 10 capsules were emptied and finely powdered. The powder amount equivalent to $20.0 \mathrm{mg}$ of lansoprazole was transferred to a $100 \mathrm{~mL}$ volumetric flask, stirred for $10 \mathrm{~min}$ with $0.1 \mathrm{M} \mathrm{HCl}$ and then diluted to volume with $0.1 \mathrm{M} \mathrm{HCl}$. The solution was then filtered through $0.2 \mu \mathrm{m}$ cellulose acetate syringe filters. The filtrate was used to prepare different concentrations within the linearity range by proper dilution with $0.1 \mathrm{M} \mathrm{HCl}$. Samples to be analyzed by the HPLC were diluted by the internal standard solution.

2) Spiked plasma samples (for HPLC method only): Plasma samples were supplied by the Blood Bank (Amman) and stored as soon as possible at $-4^{\circ} \mathrm{C}$. After being thawed, known amounts of the drug under investigation were added to drug-free plasma (100-300 $\mu \mathrm{L}$ of the 1000 $\mu \mathrm{g} / \mathrm{mL}$ per $0.5 \mathrm{~mL}$ plasma). After that, $1 \mathrm{~mL}$ of water was added. The mixture was vigorously mixed in a tube for $1 \mathrm{~min}$ and then allowed to stand for $10 \mathrm{~min}$. This mixture was applied to a solid phase extraction cartridge ( $1 \mathrm{~g}$ of $\mathrm{C} 18$, obtained from Supelco Company) that had previously been activated with $1.0 \mathrm{~mL}$ of methanol followed by $1.0 \mathrm{~mL}$ of water. The cartridge was then washed with $2.0 \mathrm{~mL}$ of water and then with $1.0 \mathrm{~mL}$ of $40 \%$ methanol in water. Lansoprazole was then eluted with $2.0 \mathrm{~mL}$ of methanol. The solvent was then evaporated to dryness under gentle stream of nitrogen and the residue was re-dissolved in $5 \mathrm{~mL}$ of the internal standard solution and injected onto the HPLC column.

3) Recovery: The recovery following the extraction procedure was determined by comparing the peak areas of blank plasma sample extracted according to the above procedure with those of non-extracted control samples. The control samples were prepared by mixing solutions containing the same amounts of the drug (same concentrations added to plasma samples) was directly injected into the HPLC apparatus. 


\section{Results and Discussion}

\subsection{Development of the Methods}

\subsubsection{FIA Method}

The proposed FIA method allows a rapid and economical quantitation of lansoprazole in pharmaceutical formulations without any time-consuming sample preparation. It was found that NBS can oxidize lansoprazole in an acidic medium. In addition, it reacts immediately with chloranilic acid (CAA) in an acidic medium to bleach out its purple color. Therefore, after the oxidation of lansoprazole by NBS, the excess NBS was reacted with the CAA. The absorption spectrum of the remaining CAA which has a maximum absorption at $530 \mathrm{~nm}$ is recorded. Consequently, the different parameters affecting the oxidation reaction, and hence the subsequent determination were optimized.

The effect of the acidity on the completeness of the reaction, and consequently on the analytical signal, was studied over acidic and basic $\mathrm{pH}$ ranges. Different buffer solutions (acetate, citrate, borate and phosphate) were used as carriers. No significant influence for the buffer type on the analytical signal was observed. However, all results indicated that the reaction was better carried out in an acidic medium. Therefore, the reactions were carried out using different types of acids with different concentrations as carriers. Hydrochloric acid gave the best results, and therefore, was used for further investigations. Gradual increase in the analytical signal was observed upon increasing the $\mathrm{HCl}$ concentration. However, the stability of the baseline and consequently the reproducibility of the results were significantly reduced at higher $\mathrm{HCl}$ concentrations. Therefore, $0.2 \mathrm{M} \mathrm{HCl}$ was used as a carrier as it gives reasonable sensitivity and baseline stability.

The influence of changing CAA concentration on the analytical signal was studied at different concentrations of NBS (0.2-1.5 mM). Fixed volumes $(100 \mu \mathrm{L})$ of lansoprazole were injected into the $\mathrm{HCl}$ stream (Figure 1). Maximum analytical signals were obtained when the concentration of CAA was $1.0 \mathrm{mM}$. In addition, different concentrations of NBS were tested. However, for all NBS and CAA combinations tested, best results were obtained when the NBS-to-CAA ratio was $1: 1$. Furthermore, maximum analytical signal was obtained when 1.0 $\mathrm{mM}$ NBS was used. Concentrations that are higher than $1.0 \mathrm{mM}$ for NBS were not tested due to its low solubility. Therefore, 1.0 mM NBS was used throughout this work.

The effect of the reagents flow rate was studied keeping other conditions constant, over the range 1.0-4.8 $\mathrm{mL} / \mathrm{min}$, with equal flow in the three channels. The highest signals were obtained when the total flow rate was 3.0 $\mathrm{mL} / \mathrm{min}$. At higher flow rates, irreproducible results and considerable decrease in the analytical signal was observed. A flow-rate of $1.0 \mathrm{~mL} / \mathrm{min}$ for each channel was chosen as a compromise between the peak shape, the sen- sitivity and the sampling time. With a flow rate of 1.0 $\mathrm{mL} / \mathrm{min}$, the analysis time for one injection was less than 50 seconds and the sample throughput was over 70 samples/h.

Two reaction coils were used in the manifold, as shown in Figure 1. The first coil (RC1), in which the oxidation of the drug by NBS took place, was changed over the range 30 to $150 \mathrm{~cm}$. A considerable increase in the analytical signal was observed upon increasing the coil length up to $120 \mathrm{~cm}$, and started to decrease after that. This indicates that the reaction between the drug under investigation and NBS was fast, and a further increase in the reaction coil length resulted in a significant peak broadening and a longer time to go back to the baseline. Therefore, the length of the first reaction coil (RC1) was chosen to be $120 \mathrm{~cm}$ to ensure high sensitivity and a high measurement rate. Similarly, a significant change in the analytical signal was observed when the second reaction coil (RC2) was increased from 30 to 150 $\mathrm{cm}$. Maximum analytical signals were obtained when $\mathrm{RC} 2$ was $50 \mathrm{~cm}$.

In order to investigate the influence of the injected sample volume on the analytical signal, different lengths of the sample loop were installed on the injector and tested. As expected, an increase in the injected sample volume leads to an increase in the peak height. Consequently, the sensitivity of measurements could be improved by increasing the sample volume. However, increasing the sample volume lead to an increase in the peak width and time for the signal to retain to the baseline. Thus, a $100 \mu \mathrm{L}$ volume was chosen that produced a reasonable sensitivity and sampling rate.

\subsubsection{HPLC Method}

Chromatographic separation of lansoprazole from its degradation products was carried out using Novapak C18 column. The chromatographic conditions were adjusted in order to provide a good performance of the assay. Several mobile phases were prepared and tested in order to achieve these requirements. Mobile phase selection was based on peak parameters (symmetry, tailing), run time, easy of preparation and cost. In these trials the separation time was long ( $>10 \mathrm{~min}$ ) with a considerable tailing in lansoprazole peak. Therefore, in order to minimize the analysis time and to improve the separation efficiency, triethylamine (TEA) was added to the mobile phase in different ratios. Figure 2 shows a typical chromatogram obtained from the analysis of a standard and a sample solution of lansoprazole using the proposed method. As shown, the lansoprazole peak is symmetrical and free from any chemical interference.

\subsection{Validation of the Proposed Methods}

The methods were validated according to International Conference on Harmonization guidelines [14] for valida- 


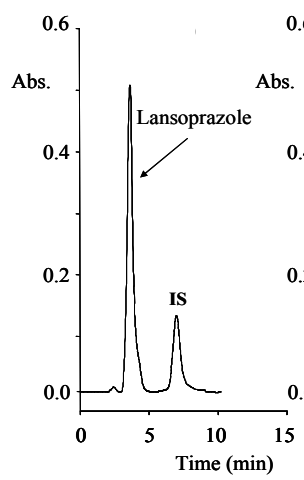

(a)

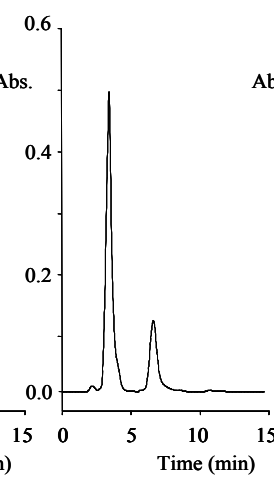

(b)

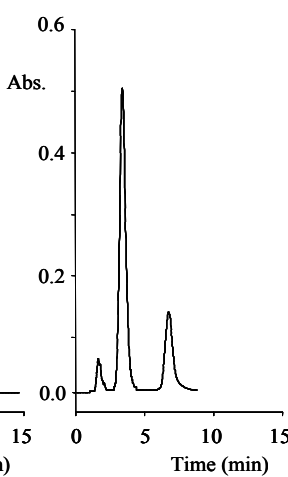

(c)
Figure 2. (a) Chromatograms for pure lansoprazole; (b) one of the commercial product, Lansozole; (c) plasma extract Concentration of lansoprazole in samples was $75 \mu \mathrm{g} / \mathrm{mL}$.

tion of analytical procedures. Analysis of variance (ANOVA) was used to verify the validity of the methods.

\subsubsection{FIA Method}

The calibration curve for the determination of lansoprazole was obtained under the optimum conditions. The calibration graph was constructed by plotting concentration versus peak area and showed good linearity in the 5$150 \mu \mathrm{g} / \mathrm{mL}$ range. The representative linear equation was $\mathrm{Y}=8.62 \times 10^{-4} \mathrm{X}+1.70 \times 10^{-3}$, with a correlation coefficient $(r=0.9988)$ highly significant for the method.

The limit of detection (LOD) and limits of quantitation (LOQ) were determined on the basis of response and slope of the regression equation. The LOD and LOQ were found to be 5.0 and $16.4 \mu \mathrm{g} / \mathrm{mL}$, respectively indicating a high sensitivity of the method.

The intra-day (within-day) precision was evaluated by replicate analysis of lansoprazole within the linearity range at different time intervals. Three measurements were taken within the same day. Each time, a new calibration graph was constructed. The result obtained shows RSD of $1.7 \%$ indicating good intra-day precision. Similarly, the inter-day (different days) precision was evaluated on different days (3 days). The results indicated high precision, as the percent RSD was $2.3 \%$. Figure 3 shows the flow signals for the calibration graph and reproducibility test. The reproducibility of the peak height was very good and the relative standard deviation of 10 injections was $1.4 \%$ (Figure 3(b)).

The accuracy of the proposed FIA method was determined by analyzing lansoprazole in synthetic mixtures. Percentage recovery from synthetic mixtures was determined by comparing the peak areas obtained from common excipients spiked with known amounts of lansoprazole. Synthetic mixtures containing different concentrations of lansoprazole, in the presence of more than 100 folds of common additives were prepared. The additives that have been used in this study are; sodium saccharide, and citric acid. The undissolved material was filtered off

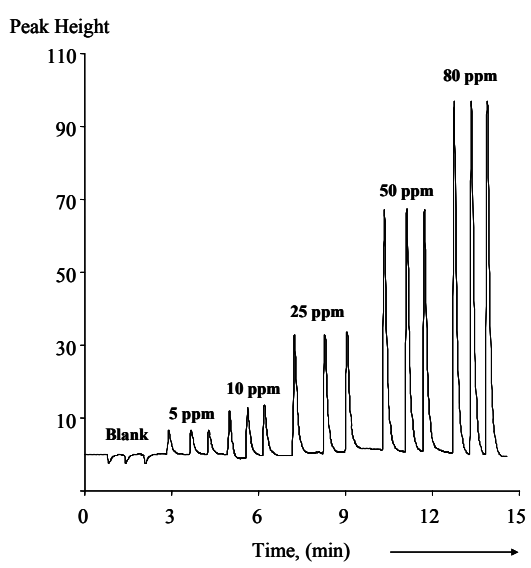

(a)

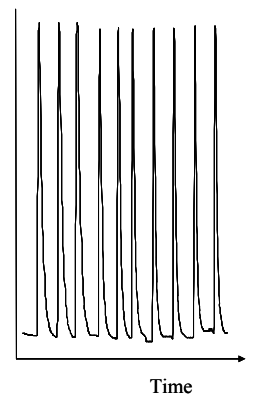

(b)
Figure 3. Flow signals for the calibration graph and reproducibility test of lansoprazole.

Table 1. Recovery of lansoprazole from synthetic mixtures and human plasma samples.

\begin{tabular}{cccc}
\hline Sample & $\begin{array}{c}\text { Added } \\
(\mu \mathrm{g} / \mathrm{mL})\end{array}$ & \multicolumn{2}{c}{$\%$ Recovery $\pm \mathrm{SD}(\mathrm{n}=5)$} \\
\hline \multirow{3}{*}{ Synthetic } & & FIA & HPLC \\
& 40 & $102.3 \pm 1.3$ & $101.2 \pm 0.9$ \\
& 80 & $97.8 \pm 0.7$ & $99.7 \pm 0.6$ \\
Plasma & 120 & $98.6 \pm 0.8$ & $97.4 \pm 2.4$ \\
& 20 & & $95.51 \pm 3.2$ \\
& 50 & & $96.9 \pm 2.0$ \\
& 100 & & $97.3 \pm 1.1$ \\
\hline
\end{tabular}

before injection. The results obtained were compared with expected values and presented in Table 1. No significant changes were observed in the results and recoveries close to $100 \%$ were achieved at all concentrations and the RSD does not exceed $2.5 \%$. The closeness of the results to the label claim supports the accuracy of the method.

\subsubsection{HPLC Method}

The calibration curves for lansoprazole were constructed by plotting concentration versus peak area and showed good linearity in the $2.0-120 \mu \mathrm{g} / \mathrm{mL}$ range. The representative linear equation was $\mathrm{Y}=4.90 \times 10^{-3} \mathrm{X}+1.10 \times$ $10^{-3}$, with a correlation coefficient (0.9993) highly significant for the method. Figure 2 shows HPLC chromatograms for $75 \mu \mathrm{g} / \mathrm{mL}$ of (a) lansoprazole standard, (b) commercial product, lansazole, (c) plasma extract. As shown, excipients used as additives in pharmaceutical formulations did not interfere in the proposed procedures.

The LOD and LOQ were found to be 2.0 and $7.0 \mu \mathrm{g} / \mathrm{mL}$, respectively indicating a high sensitivity of the method. The precision of the method was determined by repeatability (intra-day) and (inter-day) and was expressed as $\%$ RSD of a series of measurement. The result obtained 
shows RSD of $0.83 \%$ indicating good intra-day precision. Inter-day variability was calculated from assays on 3 days and shows a mean RSD of $1.85 \%$.

The accuracy of the HPLC method was determined by analyzing lansoprazole in synthetic mixtures. Same procedure describe for the FIA was followed. However, all samples in this case were diluted by the internal standard solution. The average recovery was close to $100 \%$, indicating an agreement between the true value and the value found (Table 1).

The specificity was determined for the HPLC method. Sample solutions of lansoprazole were submitted to accelerated degradation by heat $\left(70^{\circ} \mathrm{C}\right.$ for $\left.1.0 \mathrm{~h}\right)$, addition of $1.0 \mathrm{M} \mathrm{HCl}$, and oxidation by NBS, in order to verify that none of the degradation products of the analyte interfered with the quantitation of drug. The described HPLC method is specific. No interfering peaks were observed in degraded solutions and the degradation products were well resolved from the parent lansoprazole peak (Figure 4).

The robustness of the HPLC method was determined by analysis of samples under a variety of conditions such as small changes in the $\mathrm{pH}(3.7-4.3)$ and in the percentage of acetonitrile (55-65\%) in mobile phase and changing the column. The effect on retention time and peak parameters were studied. The method was found to be robust when the column and the mobile phase were varied. During these investigations, the retention times were modified, however the area and symmetry of peaks were conserved.

\subsection{Applicability of the Proposed Methods}

\subsubsection{FIA Method}

In order to evaluate the applicability of the proposed FIA method to routine pharmaceutical analysis, commercial pharmaceutical products containing lansoprazole were analyzed. Results obtained are summarized in Table 2. As shown, the recoveries are excellent and were above $95 \%$. The closeness of the results to the label claim supports the accuracy of the method.

The applicability of the proposed FIA method for plasma samples was also investigated. Plasma samples

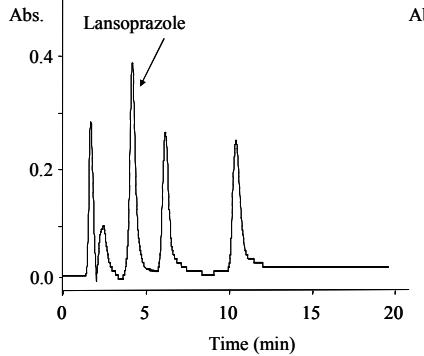

(a)

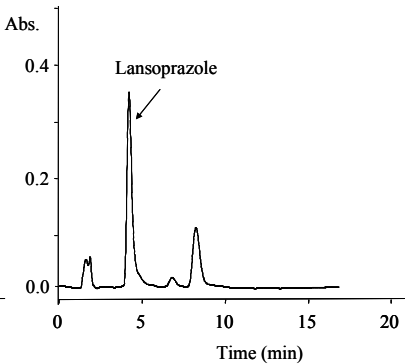

(b)
Figure 4. Chromatograms of (a) acid degraded lansoprazole and (b) NBS oxidized lansoprazole.
Table 2. FIA and HPLC results for the analysis of lansoprazole in pharmaceutical preparations.

\begin{tabular}{cccc}
\hline \multirow{2}{*}{$\begin{array}{c}\text { Trade Name \& } \\
\text { Labeled Claim }\end{array}$} & $\begin{array}{c}\text { Taken } \\
(\mu \mathrm{g} / \mathrm{mL})\end{array}$ & \%IA & HPLC \\
\cline { 3 - 4 } & 40.0 & $101.7 \pm 4.8$ & $101.2 \pm 0.2$ \\
& 80.0 & $99.3 \pm 0.3$ & $100.5 \pm 1.9$ \\
$\begin{array}{c}(\mathrm{Lansazol}) \\
(15 \mathrm{mg} / \mathrm{Cap})\end{array}$ & 120 & $97.8 \pm 0.6$ & $100.5 \pm 0.9$ \\
\hline $\begin{array}{c}\text { (Lansomid) } \\
(15 \mathrm{mg} / \text { Cap })\end{array}$ & 80.0 & $101.3 \pm 1.8$ & $102.2 \pm 2.4$ \\
& 120.0 & $96.5 \pm 1.7$ & $100.7 \pm 1.9$ \\
\hline
\end{tabular}

were spiked by different concentrations of lansoprazole. All samples were cleaned up by solid phase extraction in a similar manner to that followed before. Injection of these samples gave higher absorbance readings than the corresponding standards containing same amounts of lansoprazole. This means that the plasma components have interfered with the analysis. Precisely, most of the NBS have been consumed by the plasma components. These finding indicate that the proposed FIA method is not applicable for the determination of lansoprazole in human plasma.

\subsubsection{HPLC Method}

To evaluate the general applicability of the HPLC method, lansoprazole was analyzed in different sample matrices. The accuracy of this method was validated by the analysis of these drugs in commercial pharmaceutical formulations and spiked plasma samples. Results are summarized in Tables 1 and $\mathbf{2}$. For the pharmaceutical products, the recoveries were satisfactory and close to $100 \%$. The closeness of the results obtained to the label claimed supports the accuracy of the method. No interferences were observed from any additives in any of the products analyzed.

The proposed HPLC procedure was further validated using spiked human plasma samples. In all cases, no interferences from the indigenous plasma components were observed (Figure 2(c)). The recoveries were in the range $95-97 \%$. The simplicity of the proposed extraction procedure and the high extraction efficiency are among the essential features of the proposed HPLC method, making this method suitable for routine, efficient and fast extraction of lansoprazole from complex matrices.

\subsubsection{Comparison of the FIA Results with the HPLC Results}

The proposed analytical methods were compared using statistical analysis. The student's t-test was applied and does not reveal significant difference between the experimental values obtained in the sample analysis by the 
two methods. The calculated t-value (1.18) was found to be less than the tabulated t-value $(2.2)$ at $5 \%$ significance level.

\subsection{Conclusions}

The determination of lansoprazole in pharmaceutical products can be performed by flow injection spectrophotometric analysis with a relative standard deviation of $<5 \%$ and a rate of at least 70 samples per hour. The developed FIA method is accurate, precise, simple, and economic in consumption of samples and reagents, reproducible and can be applied for the determination of these drugs in different pharmaceutical products.

The HPLC assay of lansoprazole has been shown to be of general applicability to commercially available products and spiked human plasma samples. It could be used for the assay determination in the range of $2.0-120 \mu \mathrm{g} / \mathrm{mL}$ with a relative standard deviation of $<3 \%$. The method is accurate, precise and specific. The described HPLC assay can be easily applied for the quantification of the degradation products.

\section{Acknowledgements}

The financial support from Yarmouk University is gratefully acknowledged. The authors would like to thank Al-Hikma pharmaceuticals, Amman-Jordan, for providing the standard and the excipients.

\section{References}

[1] J. M. Ritter, L. D. Lewis and T. G. K. Mant, "A Textbook of Clinical Pharmacology," 4th Edition, Arnold Ltd., London, 1999.

[2] "The United States Pharmacopoeia," 27th Edition, "National Formulary 22," Asian Edition, Rockville, 2004.

[3] T. Uno, N. Yasui-Furukori, T. Takahata, K. Sugawara and T. Tateishi, "Determination of Lansoprazole and Two of its Metabolites by Liquid-Liquid Extraction and Automated Column Switching High-Performance-Liquid Chromatography: Application to Measuring CYP2C19 Activity," Journal of Chromatography B, Vol. 816, No. 1-2, February 2005, pp. 309-314.

[4] H. Katsuki, A. Hamada, C. Nakamura, K. Arimori and M. Nakano, "High-Performance Liquid Chromatographic Assay for the Simultaneous Determination of Lansoprazole Enantiomers and Metabolites in Human Liver Mi- crosomes," Journal of Chromatography B, Vol. 757, No. 1, pp. 127-133.

[5] Z. A. El-Sheref, A. O. Mohamad, M. G. El-Baradicy and M. F. El-Tarras, "Reversed-Phase High Performance Liquid Chromatographic Method for the Determination of Lansoprazole, Omeprazole and Pantoprazole Sodium Sesquihydrate in Presence of Their Acid-Induced Degradation Products," Chemical and Pharmaceutical Bulletin, Vol. 54, No. 6, June 2006, pp. 814-818.

[6] M. Miura, H. Tada and T. Suzuki, "Simultaneous Determination of Lansoprazole Enantiomers and their Metabolites in Plasma by Liquid Chromatography with Solid-Phase Extraction," Journal of Chromatography B, Vol. 804, No. 2, May 2004, pp. 389-395.

[7] A. Avgerinos, T. Karidas, C. Potsides and S. Axarlis, "Determination of Lansoprazole in Biological Fluids and Pharmaceutical Dosage by HPLC," European Journal of Drug Metabolism and Pharmacokinetics, Vol. 23, No. 2, 1998, pp. 329-332.

[8] M. F. Tutunji, A. M. Qaisi, B. El-Eswed, L. F. Tutunji, "An in Vitro Investigation on Acid Catalyzed Reactions of Proton Pump Inhibitors in the Absence of an Electrophile, International Journal of Pharmaceutics, Vol. 323, No. 1-2, October 2006, pp. 110-116.

[9] A. Radi, "Adsorptive Stripping Square-Wave Voltammetric Study of the Degradation of Lansoprazole in Aqueous Solutions, Microchemical Journal, Vol. 73, No. 3, December 2002, pp. 349-354.

[10] C. Yardimci and N. Ozaltin, "Electrochemical Studies and Differential Pulse Polarographic Analysis of Lansoprazole in Pharmaceuticals," The Analyst, Vol. 126, No. 3, March 2001, pp. 361-366.

[11] N. Rahman, Z. Bano, S. N. Azmi, M. Kashif, "A Kinetic Spectrophotometric Method for the Determination of Lansoprazole in Pharmaceutical Formulations," Journal of the Serbian Chemical Society, Vol. 71, No. 10, 2006, pp. 1107-1120.

[12] D. Yeniceli, D. Dogrukol-Ak and M. Tuncel, "Determination of Lansoprazole in Pharmaceuticals Capsules by Flow Injection Analysis Using UV-Detection," Journal of Pharmaceutical and Biomedical Analysis, Vol. 36, No. 1, September 2004, pp. 145-148.

[13] N. Ozaltin, "Determination of Lansoprazole in Pharmaceutical Dosage Forms by Two Different Spectroscopic Methods," Journal of Pharmaceutical and Biomedical Analysis, Vol. 20, No. 3, July 1999, pp. 599-606.

[14] "Validation of Analytical Procedures: Methodology," Proceedings of the International Conference on Harmonisation of Technical Requirements of Registration of Pharmaceuticals for Human Use, Geneva, 1996. 